\section{Cureus}

Received 04/09/2019

Review began 04/13/2019

Review ended 05/10/2019

Published 05/31/2019

\section{(c) Copyright 2019}

Gander et al. This is an open access article distributed under the terms of the Creative Commons Attribution License CC-BY 3.0., which permits unrestricted use, distribution, and reproduction in any medium, provided the original author and source are credited.

\title{
Examining the Importance of Family History in Pediatric Behavioural Referrals
}

\author{
Sarah Gander ${ }^{1}$, Sarah A. Campbell ${ }^{2}$, Kathryn E. Flood ${ }^{1}$, Emma Grace Crowley ${ }^{1}$ \\ 1. Pediatrics, Saint John Regional Hospital, Saint John, CAN 2. Pediatrics Research, Saint John Regional \\ Hospital, Saint John, CAN
}

$\square$ Corresponding author: Kathryn E. Flood, kathryn.flood@horizonnb.ca Disclosures can be found in Additional Information at the end of the article

\section{Abstract}

In recent years, there has been a substantial increase in the diagnosis of attention deficit hyperactivity disorder (ADHD) in children. Without appropriate management of symptoms and care, ADHD has been associated with a variety of negative child and adult outcomes.

Environmental and familial factors that may contribute to three different pediatric referral types (academic, behavioural, and attentional) associated with ADHD were examined in the current study. In total, data from 477 families who were interviewed as a part of the intake process to a pediatric clinic were included in this study. Data for the current study was extracted from the intake questionnaires and included information on family history of mental health issues, socioeconomic status, and family relationships. The sample included children between the ages of three and 17 and mostly comprised males $(n=340)$. A frequency analysis of the data demonstrated relatively high rates of mental health issues within families (61.4\%); almost half of the mothers reported some post-secondary education (46.1\%) and most reported having normal relationships with their children (mothers, 78\%; fathers, 62.9\%). Finally, three stepwise regression analyses were conducted to predict referral type. All three regressions yielded significant models. Fifteen percent of the variability of the academic referral type was predicted by being male, age at the time of referral, mother's education level, and mother's learning. The behavioural referral types were predicted by a family history of depression, being male, mother-child relationship, and age at the time of referral; these accounted for $23 \%$ of the variance. Attentional referral type was predicted only by mother-child relationship that captured $6 \%$ of the variance. Overall, this study describes a population of parents of children with academic, behavioural, and attention-related referrals to pediatrics. Results indicate that mothers have a profound influence on their child's referral types, something that may transfer into later diagnosis and perhaps prognosis. Clinicians and researchers alike should focus their efforts toward developing integrative service assessment and treatment approaches that include important people in the child's life. The implementation of Community Social Pediatrics (a streamlined, inclusive approach to care) should be considered in urban centres like this one, where referrals like this are prevalent.

Categories: Pediatrics, Psychology

Keywords: attention deficit hyperactivity disorder, attention, academic, behaviour, referrals, pediatrics, mother-child relationship, social pediatrics, attention deficit hyperactivity disorder, socioeconomic status, attention, academic, behaviour

\section{Introduction}

Attention-deficit hyperactivity disorder (ADHD) is an increasingly diagnosed behavioural disorder characterized by many symptoms including an inability to focus, increased distractibility, and forgetfulness [1]. The implications of these symptoms over time have been 
associated with educational difficulties, relationship issues, and increased engagement in risky behaviours [2]. In addition, comorbid diagnosis of other conditions (i.e., anxiety, depression, substance use, oppositional defiant disorder, and borderline personality disorder) are common with ADHD [3]. It is imperative to identify and manage these behaviours early, as a lack of treatment can lead to a poor prognosis for ADHD symptoms in adulthood. These symptoms also may be indicative of the underlying environmental or familial stressors being encountered by the child that mirror or augment ADHD symptoms, indicating that consultant pediatricians should consider and evaluate additional indicators (i.e., socioeconomic status) that may be contributing to ADHD symptomology [4].

Socioeconomic status (SES) encompasses factors such as income, education, family history, and geographic location, among others [5]. Individuals who report lower SES may have certain barriers accessing appropriate health care versus individuals with higher SES who are equipped with certain tools (e.g., education, transportation, etc.) because of their background. More specifically, children and families from vulnerable populations may not have the skills to confidently navigate the healthcare system even if they recognize a need for support. This is often due to a lack of knowledge in their rights, healthcare systems, and even normal child development [6]. Factors like low SES may be mediating delays in diagnoses for these vulnerable families, resulting in even poorer outcomes for the child.

Although somewhat heritable, ADHD severity may be influenced by an individual's early environment. Environmental risk factors include maternal pregnancy complications, low child socialization and nutritional deficits [7]. Moreover, parental mental illness and childhood psychiatric conditions (e.g., ADHD) have been positively associated. That is, children of parents with certain mental illnesses (i.e., bipolar disorder, schizophrenia, depression, alcohol- or substance-use disorder) may be more susceptible to the development of disorders like ADHD [8-13]. Recently, a study conducted by López Seco and colleagues suggested that half of the children in their sample with ADHD had at least one parent with a psychiatric history [14]. Taken together, the importance of understanding familial and social health factors may be integral to early detection and appropriately -tailored treatment of disorders like ADHD. By enhancing our knowledge of a child's familial situation, clinicians may be able to provide more targeted care and implement useful social programs. In addition, by understanding the relations between parental mental illness and child behaviour issues, we could be able to screen and treat both parents and children in tandem, which may lead to better outcomes overall.

The present study was conducted in a medium-sized Atlantic Canadian city with an urban core of approximately 70,000 residents, but providing pediatric services to a catchment of almost 300,000 . The child poverty rate in this area is one of the highest in Canada, at 30\%, almost double the national average. Additionally, half of all single-family households in NB live in poverty compared to approximately $10 \%$ of two-parent households [15]. Thus, research surrounding SES, behavioural disorders, and familial histories are timely given the situation this city is facing.

The current study examined the family mental-health histories and SES of children who have received pediatric referrals for behaviour-related issues. Through a deeper understanding of the familial demographics in this referral base, clinicians may be able to design and implement family-specific interventions, ultimately aiding in decreasing barriers to care. Furthermore, by understanding situational factors like SES that may be predictive of certain referral types early on, clinicians and other community partners may be able to target and treat vulnerable children earlier which could lead to better outcomes and less time without support and guidance.

\section{Materials And Methods}




\section{Participants}

In total, 477 families were interviewed pre-pediatric visit as a part of the intake process. Interviews were administered by a clinic nurse, with a parent or guardian of the children being referred to the clinic.

\section{Materials}

Data for the current study was extracted from comprehensive intake questionnaires developed by local pediatricians (see Appendix 1). The intake interview was administered to patient parents/guardians between the years of 2012 and 2017 and contained questions regarding medical, familial, and neurodevelopmental histories. The primary purpose of these interviews was to provide pediatricians with a comprehensive picture of a child's history and needs. Therefore, because research was not originally intended, ethical approval for secondary use of data was granted from the Horizon Health Network's Research Ethics Board. Lastly, statistical analyses were conducted using IBM SPSS statistical software, Version 22.

\section{Procedure}

The data were collected via telephone intake interviews of children being referred to pediatricians for behaviour-related referrals. Interview questions explored details pertaining to the child's medical, academic and family history and other factors. The telephone interview, conducted by a clinical nurse, was done with a primary caregiver in the child's life, such as a parent, grandparent, custodial guardian. Information from the interviews was input into a database and appropriately coded for analyses. Descriptive statistics were noted for all variables to understand the sample. Finally, three stepwise regressions were conducted to predict referral type (i.e., academic, behavioural, or attention) using SES and other familial information collected in the interviews.

\section{Results}

The current sample consisted of children ranging from three to 17 years old, 340 males (MAGE= 7.57 ) and 137 females (MAGE $=7.41)$. Over half $(62.9 \%)$ of the children being referred for behavioural, academic, or attention concerns had a family history of mental health issues. Mental illness was further categorized by disorder or illness type (Table 1). In the current sample, depression was the most prevalent followed by substance use disorder. 


\section{Cureus}

\section{Family History of Mental IIIness}

Mental Health Issues

Anxiety

Depression

ADHD

Learning Problems

Manic Depression

Schizophrenia

Bipolar Disorder

Substance Use Disorder

Anger Management Issues

\section{Percentage of Sample}

$62.9 \%$

$33.8 \%$

$49.4 \%$

$31.8 \%$

$23.2 \%$

$2.2 \%$

$10.6 \%$

$18.0 \%$

$44.9 \%$

$37.6 \%$

TABLE 1: Percentage of family histories of mental health issues by category

Factors related to SES also were collected, including levels of parental education, current employment, issues with employment and custody status. Almost half (47.8\%) of the mothers in the current sample had at least some post-secondary education, while $52.2 \%$ of mothers reported having no post-secondary education with $15 \%$ having not graduated high school. Similarly, 35.3\% of fathers had some post-secondary education, although, $21.6 \%$ indicated they had not graduated high school. Furthermore, at the time of intake, 36.2\% of mothers and $21 \%$ of fathers were unemployed, and $24.4 \%$ of mothers and $24.7 \%$ of fathers reported issues with maintaining employment. Most of the children were in the custody of both parents (44.5\%), although $41.8 \%$ were in the custody of their mother only, $4.5 \%$ father only, $7.1 \%$ shared parental custody, and $0.9 \%$ other.

The intake interview also inquired about the perceived relationship between parents and children, while many families reported relatively normal relationships; however, there were some levels of indifference and conflict recorded as well (Table 2). The frequency of family shared meals was relatively high, $78.0 \%$ suggesting that they usually shared a meal as a family. Lastly, $24.8 \%$ of families reported receiving some type of parental training. 


\section{Cureus}

\begin{tabular}{|c|c|c|}
\hline & Mother-Child Relationship & Father-Child Relationship \\
\hline Normal & $84.4 \%$ & $69.7 \%$ \\
\hline Conflicted & $14.5 \%$ & $13.6 \%$ \\
\hline Indifferent & $1.1 \%$ & $16.6 \%$ \\
\hline
\end{tabular}

TABLE 2: Percentage of parent-child relationship types in the current sample

\begin{tabular}{|c|c|c|c|c|c|}
\hline & \multicolumn{4}{|c|}{ Academic Referral } & \multirow[b]{2}{*}{$p$} \\
\hline & $\mathrm{R}$ & B & $\mathrm{t}$ & F change & \\
\hline STEP 1 & & & & & $\mathrm{R}^{2}=.06$ \\
\hline Gender & $0.26^{\star \star}$ & 0.26 & 3.18 & 10.11 & 0.002 \\
\hline STEP 2 & & & & & $\Delta R^{2}=.09$ \\
\hline Age at Referral & $0.31^{\star \star}$ & 0.03 & 2.05 & 7.29 & 0.001 \\
\hline STEP 3 & & & & & $\Delta R^{2}=.12$ \\
\hline Mother's Education & $0.35^{\star}$ & 0.06 & 2.01 & 6.31 & 0.033 \\
\hline STEP 4 & & & & & $\Delta R^{2}=.15$ \\
\hline Mother's Learning & $0.39^{*}$ & 0.23 & 2.27 & 6.17 & \\
\hline
\end{tabular}

\section{TABLE 3: Stepwise regression predicting academic referral type}

${ }^{* *} p<0.01,{ }^{*} p<0.05$

Finally, three stepwise regressions were conducted entering SES and relationship variables into the regression equation to predict referral type (i.e., academic, behavioural, and attention).

Stepwise regressions were performed as exploratory analyses given the retrospective use of the initial data, to provide a base for future prediction equation modelling by eliminating variables that were not predictive of referral type. Significant models were found for all referral types. Four significant models were found for academic referral type, although the overall model accounted for $15 \%$ of the variance in academic referrals $(F(4,134)=6.17), p<0.0001)$. That is, being male, age at the time of referral, mothers with lower education levels and mothers who reported prior learning issues were predictive of academic referral type (Table 3).

(*)

Stepwise analyses also suggested that these factors may be predictive of behavioural referral types. Four significant models emerged, model 4 accounted for $23 \%$ of the variability in behavioural referral type. A positive family history of depression, being male, an "abnormal" mother-child relationship, and a younger age at referral were predictive of a behavioural 


\section{Cureus}

referral $(F(4,136)=10.26), p<0.0001)($ Table 4$)$.

\begin{tabular}{|c|c|c|c|c|c|}
\hline & \multicolumn{4}{|c|}{ Behavioural Referral } & \multirow[b]{2}{*}{$\mathrm{p}$} \\
\hline & $\mathrm{R}$ & B & $\mathrm{t}$ & F change & \\
\hline STEP 1 & & & & & $R^{2}=0.08$ \\
\hline Family history of depression & $0.30^{* \pi}$ & 0.29 & 3.72 & 13.87 & 0.000 \\
\hline SIEP 2 & & & & & $\Delta R^{20}=0.15$ \\
\hline Gender & $0.39^{* \pi}$ & -0.27 & -3.25 & 12.11 & 0.000 \\
\hline STEP 3 & & & & & $\Delta R^{2}=0.20$ \\
\hline Relationship with Mother & $0.44^{\star \star}$ & 0.22 & 2.73 & 11.37 & 0.000 \\
\hline STEP 4 & & & & & $\Delta R^{2}=0.23$ \\
\hline Age at Referral & $.48^{\star \star}$ & -0.03 & -2.39 & 10.26 & 0.000 \\
\hline
\end{tabular}

\section{TABLE 4: Stepwise regression predicting behavioural referral type}

${ }^{* *} p<0.01,{ }^{*} p<0.05$

Only one model emerged to predict attention referral types, that is, mother-child relationship (Table 5). That is, relationship with mother accounted for $6 \%$ of the variance in attention type referrals $(F(1,137)=8.78), p<0.004)$.

\begin{tabular}{|c|c|c|c|c|c|}
\hline & \multirow[b]{2}{*}{$\mathrm{R}$} & \multicolumn{3}{|c|}{ Attention Referral } & \multirow[b]{2}{*}{$p$} \\
\hline & & B & $\mathrm{t}$ & F & \\
\hline & & & & & $\mathrm{R}^{2}=0.06$ \\
\hline Relationship with Mother & $0.25^{* \star}$ & -.027 & -2.96 & 8.78 & 0.004 \\
\hline
\end{tabular}

\section{TABLE 5: Stepwise regression predicting attention referral types}

${ }^{* *} p<0.01$

\section{Discussion}

The number of children who are experiencing issues related to behaviour, attention, academic, and social difficulties is reaching a critical point [16]. When these children are referred to pediatricians and other clinicians, they often encounter long wait times and a fragmented system of services and resources. Service providers are ill-equipped to consider the complex 
social and family histories of these children and address these challenges in their assessments and care pathways. Through developing a better understanding of the underlying issues that contribute to children's difficulties, we hope to identify better treatment paths and to more adequately address the full complement of needs that are relevant to children and their families, discussed further below.

Overall, this study described a population of parents of children with academic, behavioural, and attention-related referrals to pediatricians. Being male was predictive of increased academic and behavioural referral types, which is unsurprising given most of the current sample was composed of male referrals and meta-analytic results that have suggested males present more external problems related to disorders like ADHD (i.e., hyperactivity, inattention, and impulsivity) [17]. Younger age also was a significant predictor of behavioural referral type, though it is important to note that despite ages ranging from 3 to $17,78.8 \%$ of the sample was under the age of nine, which may be due to adolescents being referred to other types of clinicians (e.g., psychiatrists, psychologists, social workers, etc.).

In Canada, 50\% of individuals report issues with mental health at some point in their lives [18]. Therefore, the fact that $62.9 \%$ of families in this study have a family history of mental illness is not necessarily unusual. Future research could include a more in-depth screening to identify the number of children with parental mental health issues, given the current data focused on entire family history. This aligns with previous research suggesting that this population often require services from other allied health professionals [19], suggesting that these cases are complex and require the application of a more coordinated assessment method.

The role of mother-child relationships have long been identified as a key factor in child development and beyond. Studies have demonstrated that nurture plays a key role in many aspects of development later informing adult experiences (e.g., future relationships, health outcomes, and life satisfaction [20-24]). Impaired mother-child relationship was a significant predictor of attention and behaviour-related referrals in the current study. ADHD symptoms have been linked to mother-child relationship before, one study suggested that there was an association with increased ADHD symptoms and hostility in mother-child relationships, controlling for biological factors [24]. Further, one study suggested that mothers with depression may have children diagnosed with ADHD who demonstrate increased behavioural issues over and above mothers of children with ADHD who report lower levels of depressive symptoms [25]. These are just a few of the associations found between mothers and their child's behavioural and attention-related issues, warranting further investigation. More specifically, future research should note the child's later diagnosis after the referral process in order to make additional inferences about these relations. Finally, these results do not necessarily suggest that the mother-child relationship is more important than the relationship between the father and their child. Conversely, it should be noted that $41.8 \%$ of the current sample was in the custody of their mothers only, while only $4.5 \%$ were in the custody of their father's only; given these results, direct comparisons cannot be made.

Although mother-child relationship did not emerge as a significant predictor of academic referral types, it is interesting to note that the mother's education and learning history did emerge as predictors of academic referrals. This result suggests that a mother's history may impact a child's performance over and above other factors, specifically, their father's history. It is clear that mothers have a significant impact on their children's referral type and the relation with the child's eventual or future diagnoses within the current sample should be explored. Perhaps, future research should focus on the importance of parent-child relationships and other familial and environmental factors when examining pediatric referral types and potential correlates, specifically how these are measured. 
The current study was limited in that it was conducted using secondary data developed for clinical, and not research, purposes. Further to the point, the use of stepwise regression analyses is not ideal given the increased number of variables entered into the equation to find the model best fit; however, this method may provide future researchers with knowledge to make informed a priori hypotheses and ultimately capture a larger amount of variance in referral types using these (e.g., parent-child relationship, family history of depression, etc.) and other predictors.

Given the current results, another limitation of this retrospective data was the operationalization of certain variables like parent-child relationship. In the current study, parent-child relationship was measured by simply asking if the child's relationship with their mother or father (from the guardian's perspective) was "normal, conflicted, or indifferent", although this is useful for clinical purposes, it would be more valuable to use psychometrically validated measures in the future. For example, measuring a child's attachment (i.e., bonds formed with certain individuals like caregivers that inform a variety of an individual's behaviours throughout their lifespan) with their caregiver may better illustrate the influence of parent-child relationships on pediatric referral types [20-23]. Family and child history also may be better captured through a child and even a guardian's Adverse Childhood Experience (ACE) scores, simultaneously guiding the clinician to alternative treatment pathways [25]. Regardless, this intake interview has proven to be a valuable tool for informing future research and we feel can now be refined further and paired with psychometric tools for optimal results.

It is beneficial for pediatric clinicians to understand markers of some of the more common conditions that parents and guardians reported (i.e., depression, anger management, substance dependence, and anxiety) but also, how the presence of these conditions in caregivers may be affecting the child's health and well-being. Further, a guardian suffering from mental illness may make child treatment plans increasingly difficult for caregivers to effectively navigate with their child. By understanding mental health concerns in families, we can introduce familybased solutions to improve compliance.

Increasingly, there are many examples in practice throughout Canada and globally that stress the importance of the psychosocial model and incorporating the needs and strengths of the family in planning for care and treatment. The Community Social Pediatrics (CSP) model stresses the importance of coming together around the child as caregivers to support the child to realize their full potential [26]. This study illustrates the importance of engaging caregivers in the optimization of their own mental health to meet their child's needs. This includes promoting the importance of caregiver-child relationships to provide a safe and nurturing environment for development. It recognizes there may be an early warning for children at risk for attention and behavioural problems and that early intervention will likely provide better outcomes.

A CSP approach offers a streamlined pathway to care for children, specifically those from low SES families where communication across services tends to break down, creating further problems including access to timely care and misdiagnosis. CSP models have demonstrated positive results wherein children demonstrate improvements in their academic grades and overall sense of well-being. It is well-documented children facing social disparities, trauma and toxic stress will experience a disproportionate number of negative physical and mental health outcomes across their lifespan [6,26-29].

\section{Conclusions}

Future researchers and clinicians should focus on this model to build a team that would perform a comprehensive assessment of the child and build a care and treatment plan that considers the entire situation of the child. The implications this approach may have on these 


\section{Cureus}

children may involve not only short-term benefits (i.e., better health, academic success) but a more inclusive picture of the child's risk factors (e.g., ACEs), allowing professionals to intervene in a timely manner and ultimately decrease the child's use of the system throughout their lifespan.

\section{Appendices}

\section{Name:}

Date:

Pediatrician:

DOB $(\mathrm{dd} / \mathrm{mm} / \mathrm{yy})$

Medicare:

School:

Grade:

Teacher:

Home meds:

Pharmacy:

Insurance:

\section{Email}

Phone

Mother

Father

Stepfather

Custodial Parent

\section{SNAP Forms}

Informant

Brother(s)

Sister(s)

Dept of Social Development

Foster Care

Referral Source

By:

Concerns:

Resources in place

Name

\section{Dates involved}

Family Doctor

Psychologist 


\section{Cureus}

\section{PsychEd Assessment}

Mental Health Services

Parent Training

Addictions Counselling

Anger Management

Alternative Therapy

Early Intervention

OT

PT

SLP

\section{Pregnancy}

Maternal Age:

Gestation at Delivery

EGA: $\quad$ Birth Weight:

Hypertenison

Diabetes

Infections

Cig/EtOH/drugs

Gestational Problems

Postnatal complications

Development

Gross Motor

Fine Motor

Language

Socialization

Vision/Hearing

Dental

Other

\section{Past Medical History}

No

Past Medical Diagnosis

Admissions 


\section{Cureus}

Surgery

Psychiatric

Allergies

Immunizations

Other

\section{Review of Systems}

Skin (eczema, rash)

ENT (ear, throat, nose)

Respiratory (day or night cough)

Gastrointestinal

Genitourinary

Cardiovascular

Musculoskeletal

Hematological

Neurological

Puberty

Birthmarks/ Hair Growths

\section{Daycare/School History}

Years attended/ Concerns raised/ Teacher name

Daycare/preschool

Kindergarten

Grade 1

Grade 2

\section{Function Assessment}

Sleep, Hygiene (Bedtime, waking, sleep habits)

Toileting

Eating (family habits, meal times)

Relationship / c parents

Regular chores

Friends

Gets along well with peers 


\section{Cureus}

Homework

Learning

Written output

Participation

Academic Grades

Classroom behaviour

Adaptability

Gets along well with elders

Ability to be independent / organized

Weekly exercise

Free-time behaviour

Extracurricular activities

Sports involvement

Afterschool activity

Plays with pets

Handles being teased

Socially appropriate

Humour

Sexual behaviour

Emotional coping

Does your child have any of the following?

Shortness of breath during exercise (more than other kids their age) and without any other reason like asthma, obesity, not exercising regularly.

Poor exercise tolerance (when compared to other kids their age)

Fainting or seizure with exercise or if startled

Palpitations (skipped heartbeat) with exercise

Family members (brothers, sisters, cousins, etc.) with any of the following:

Long QT syndrome

Wolff Parkinson White syndrome

Cardiomyopathy

Heart transplant 


\section{Cureus}

Pulmonary Hypertension

Unexplained death or death by sudden drowning or car accident

Sudden infant death syndrome

Implanted defibrillator

Have you ever been told your child has:

High blood pressure on exam

Murmur heard on exam

Needed heart surgery

\section{TABLE 6: Nurse intake form}

\section{Additional Information Disclosures}

Human subjects: Consent was obtained by all participants in this study. Research Ethics Board: Horizon Health Network issued approval 10417. Animal subjects: All authors have confirmed that this study did not involve animal subjects or tissue. Conflicts of interest: In compliance with the ICMJE uniform disclosure form, all authors declare the following:

Payment/services info: All authors have declared that no financial support was received from any organization for the submitted work. Financial relationships: All authors have declared that they have no financial relationships at present or within the previous three years with any organizations that might have an interest in the submitted work. Other relationships: All authors have declared that there are no other relationships or activities that could appear to have influenced the submitted work.

\section{References}

1. American Psychiatric Association: Diagnostic and Statistical Manual of Mental Disorders . Arlington, VA; 10.1176/appi.books.9780890425596

2. Sharma A, Couture J: A review of the pathophysiology, etiology, and treatment of attentiondeficit hyperactivity disorder (ADHD). Ann Pharmacother. 2014, 48:209-25. 10.1177/1060028013510699

3. Canadian ADHD Resource Alliance: Chapter 2: Differential diagnosis and comorbid disorders . (2011). Accessed: May 17, 2019: https://caddra.ca/pdfs/caddraGuidelines2011Chapter02.pdf.

4. Roy A, Hechtman L, Arnold LE, et al.: Childhood factors affecting persistence and desistence of attention-deficit/hyperactivity disorder symptoms in adulthood: results from the MTA. J Am Acad Child Adolesc Psychiatry. 2016, 1:937-44. 10.1016/j.jaac.2016.05.027

5. Baker EH: Socioeconomic status, definition. The Wiley Blackwell Encyclopedia of Health, Illness, Behavior, and Society. Cockerham WC, Dingwall R, Quah SR, et al. (ed): John Wiley \& Sons, Ltd, New Jersey; 2014. 10.1002/9781118410868

6. Lynam MJ, Scott L, Loock C, Wong S: The RICHER social pediatrics model: fostering access and reducing inequities in children's health. Healthc Q. 2011, 14:41-56. 10.12927/hcq.2011.22576

7. Austerman J: ADHD and behavioral disorders: assessment, management, and an update from DSM-5. Cleve Clin J Med. 2015, 1:3. 10.3949/ccjm.82.s1.01

8. Bauer NS, Anand V, Carroll AE, Downs SM: Secondhand smoke exposure, parental depressive symptoms and preschool behavioral outcomes. J Pediatr Nurs. 2015, 1:227-35. 
10.1016/j.pedn.2014.06.004

9. Chang KD, Steiner H, Keiter TA: Psychiatric phenomenology of child and adolescent bipolar offspring. J Am Acad Child Adolesc Psychiatry. 2000, 39:4. 10.1097/00004583-20000400000014

10. Cheng CM, Chang WH, Chen $\mathrm{MH}$, et al.: Co-aggregation of major psychiatric disorders in individuals with first-degree relatives with schizophrenia: a nationwide population-based study. Mol Psychiatry. 2018, 23:1756-1763. 10.1038/mp.2017.217

11. Goldstein BI, Shamseddeen W, Axelson DA, et al.: Clinical, demographic, and familial correlates of bipolar spectrum disorders among offspring of parents with bipolar disorder. J Am Acad Child Adolesc Psychiatry. 2010, 49:388-396. 10.1016/j.jaac.2010.01.009

12. Kuperman S, Schlosser SS, Lidral A, Reich W: Relationship of child psychopathology to parental alcoholism and antisocial personality disorder. J Am Acad Child Adolesc Psychiatry. 1999, 38:686-692. 10.1097/00004583-199906000-00015

13. Ross RG, Compagnon N: Diagnosis and treatment of psychiatric disorders in children with a schizophrenic parent. Schizophr Res. 2001, 50:121-129. 10.1016/S0920-9964(00)00043-8

14. López Seco F, Aguado-Gracia J, Mundo-Cid P, et al.: Maternal psychiatric history is associated with the symptom severity of ADHD in offspring. Psychiatry Res. 2015:226:2, 507-512. 10.1016/j.psychres.2015.02.010

15. Human Development Council. The face of child poverty in New Brunswick . (2017). Accessed: March 3, 2019: https://0102.nccdn.net/1_5/000/000/00d/99b/CPRC2017.pdf.

16. Shaw K, Wagner I, Eastwood H, Mitchell G: A qualitative study of Australian GPs' attitudes and practices in the diagnosis and management of attention-deficit/hyperactivity disorder (ADHD). Fam Pract. 2003, 20:129-34. 10.1093/fampra/20.2.129

17. Gershon J, Gershon J: A meta-analytic review of gender differences in ADHD . J Atten Disord. 2002, 5:143-154. 10.1177/108705470200500302

18. Making the case for investing in mental health in Canada. (2013). Accessed: March 1, 2019: https://cmha.ca/about-cmha/fast-facts-about-mental-illness. .

19. Social Epidemiology. Berkman LF, Kawachi I, Glymour MM (ed): Oxford University Press, New York; 2000.

20. Bretherton I, Munholland KA: Internal Working Models in Attachment Relationships: A Construct Revisited. The Guilford Press, New York; 1999.

21. Hazan C, Shaver P: Romantic love conceptualized as an attachment process. J Pers Soc Psychol. 1987, 52:511-524.

22. Bowlby J: Attachment and Loss, Volume I: Attachment. Basic Books, New York; 1969.

23. Guarnieri S, Smorti M, Tani F: Attachment relationships and life satisfaction during emerging adulthood. Soc Indic Res. 2015, 121:3-833. 10.1007/s11205-014-0655-1

24. Harold GT, Leve LD, Barrett D, et al.: Biological and rearing mother influences on child ADHD symptoms: revisiting the developmental interface between nature and nurture. J Child Psychol Psychiatry. 2013, 54:1038-1046. 10.1111/jcpp.12100

25. Chi TC, Hinshaw SP: Mother-child relationships of children with ADHD: the role of maternal depressive symptoms and depression-related distortions. J Abnorm Child Psychol. 2002, 30:387-400. 10.1023/A:1015770025043

26. Dr. Gilles Julien Foundation: Social Pediatric Centre CPSG . (2017). Accessed: May 17, 2019: http://www.pediatriesocialegatineau.com/en/gatineau-social-pediatric-center/fondation-drgilles-julien.

27. ACE interface: the adverse childhood experiences study. (2014). Accessed: February 15, 2018: https://www.aceinterface.com/.

28. Examining the Health Disparities Research Plan of the National Institutes of Health: Unfinished Business. National Academy of Sciences, 2006.

https://www.ncbi.nlm.nih.gov/books/NBK57037/

29. Government of Canada SC: child abuse and physical health in adulthood . (2016). Accessed: February 15, 2018: https:/www150.statcan.gc.ca/n1/pub/82-003-x/2016003/article/14339eng.htm. 